\title{
Acute haemoperitoneum due to broad ligament tears and utero-ovarian vessel rupture secondary to endometriosis
}

\author{
Daksha Bhobe, Eki Emovon
}

Correspondence: Dr Daksha Bhobe, Department of Obstetrics and Gynaecology, Doncaster and Bassetlaw Hospitals NHS Foundation Trust, Armthorpe Road, Doncaster, UK, DN2 5LT; Email-dakshabangui@yahoo.co.in

Distributed under Creative Commons Attribution-Share Alike 4.0 International.

\begin{abstract}
Acute haemoperitoneum in pregnancy due to tears in broad ligament and utero-ovarian vessel rupture is quite rare. Endometriosis is an important pelvic pathology in many such cases. We present a case of primigravida, a known case of endometriosis, at 14.5 weeks of pregnancy who presented with hypovolemic shock and was found to have broad ligament tears and oozing from utero-ovarian vessels and hemoperitoneum at laparotomy. Subsequently she had a miscarriage and was found to have pelvic hematoma which was successfully managed with radiological intervention thus reducing maternal morbidity. She had a successful normal delivery 5 years later.
\end{abstract}

Keywords: Broad ligament, hemoperitoneum, uterine artery embolisation, spontaneous, tears, endometriosis.

Endometriosis is one of the important factors causing utero-ovarian vessel rupture and broad ligament tears in pregnancy. It is important to keep this in mind in cases of differential diagnosis of causes of hypovolemic shock especially if pregnancy is conceived through IVF. Treatment through interventional radiology can reduce morbidity and improves outcome if prompt laparotomy is not deemed necessary. Timely diagnosis and intervention is very crucial to avoid maternal and perinatal mortality.

\section{Case report}

We present a case of a 32 year old Afro-Caribbean lady who conceived with twins through IVF. Dating scan revealed a single live foetus of 14.5 weeks $\left(2^{\text {nd }}\right.$ vanishing twin) and $4.6 \mathrm{~cm}$ fibroid in left adnexa. Past history included severe endometriosis, trigeminal neuralgia and right sided deafness.

She presented at 16 weeks with severe abdominal pain and vomiting. There was no history of vaginal bleeding, trauma, contractions or substance use. Ultrasound showed small volume of fluid in the right flank and pelvis and a live foetus. Haemoglobin was 97 $\mathrm{gm} / \mathrm{L}$ and WBC count was $9.5 \times 10^{9} / \mathrm{L}$. Her pain settled with analgesics and she was discharged after 48 hours. She re-presented 10 hours later with acute abdominal pain and collapse. Her pulse was $98 / \mathrm{min}$ and BP was $60 / 40 \mathrm{~mm}$ of $\mathrm{Hg}$. Clinically there was rebound tenderness and guarding. Emergency laparotomy

Received: $16^{\text {th }}$ February 2017. Accepted: $16^{\text {th }}$ April 2017.

Bhobe D, Emovon E. Acute haemoperitoneum due to broad ligament tears and utero-ovarian vessel rupture secondary to endometriosis. The New Indian Journal of OBGYN. 2017; 4(1):87-9 
revealed $1500 \mathrm{ml}$ of haemoperitoneum and required 5 units of blood transfusion. Linear tears were noted on the right broad ligament with ovary extruding anteriorly. Oozing from the base of the tear was sutured with 00-PDS. The left tube and ovary were adherent to the posterior wall of the uterus. She made an uneventful recovery and was discharged on the $7^{\text {th }}$ postoperative day.

She had a miscarriage 15 days later and was transfused 3 units of blood due to falling haemoglobin of $69 \mathrm{gm} / \mathrm{L}$. She was admitted 4 days later with abdominal pain. CT scan showed large haematoma in peritoneal cavity with likely bleeding from the left adnexa. She was managed conservatively and a further 4 units of blood were transfused. Embolization of left uterine artery was performed during which, a pseudoaneurysm was seen. She made an uneventful recovery.

She was advised to consider surrogacy but conceived spontaneously 5 years later with an uneventful normal vaginal delivery.

\section{Discussion}

Haemoperitoneum in pregnancy is rare and potentially lethal condition. Presenting symptoms include abdominal pain, dizziness, hypovolemic shock and foetal compromise. Differential diagnoses include uterine rupture, abdominal pregnancy, placental abruption, HELLP, perforated appendix, rupture of broad ligament, uterine torsion, uterine vein rupture, rupture of splenic or hepatic artery aneurysms ${ }^{1}$.

Factors responsible for spontaneous rupture of uterine vessels during pregnancy include adhesions, previous uterine surgery, fibroids, arteriovenous malformation, uterine artery aneurysm, increased venous pressure(vomiting, defecation, $2^{\text {nd }}$ stage pushing), weakness of vessel wall related to endometriotic lesions, free anastomosis of uteroovarian vessels in the broad ligament and absence of valves in ovarian veins ${ }^{2,3}$.

In a review of literature by Brosens I et al ${ }^{4}$, pelvic endometriosis has been found to play important role in pathogenesis of spontaneous haemoperitoneum in pregnancy. In $90 \%$ cases, bleeding site was either posterior side of uterus or parametrium. All cases of haemoperitoneum after IVF treatment were in women with endometriosis. A more recent review by Brosens et al ${ }^{5}$ have highlighted IVF with controlled ovarian hyperstimulation and embryo transfer (COH-ET) as potential risk factor. In their review, Leone Roberti Maggiore et al ${ }^{6}$ have highlighted sustained non resolving inflammation and fibrosis in endometriosis. This could be amplified by hormone sensitive intrinsic inflammation of endometriotic lesions due to pregnancy. Intrusion of decidualised endometriotic foci into tissues can increase back pressure predisposing to rupture. Necrosis of foci of decidualised ectopic endometrium located close to dilated vessels could cause dysfunctional rupture of vessels with bleeding of unpredictable severity. According to Roche et al ${ }^{1}$, adhesions can increase the risk of rupture due to raised tension on these friable vessels due to an enlarging uterus.

In our case, there was a background of severe endometriosis with adhesions with IVF pregnancy. Enlarging uterus with adhesions could have increased the tension on the broad ligament causing tears resulting in rupture of friable utero-ovarian vessels leading to haemoperitoneum.

Spontaneous broad ligament tears or utero-ovarian vessel rupture should be considered as a differential diagnosis when a pregnant woman presents with acute abdominal pain and hemodynamic instability. Treatment would include immediate laparotomy with fluid resuscitation and blood transfusion. In our case, she re-presented with haemoperitoneum following miscarriage with evidence of pseudoaneurysm in the left uterine artery which is one of the rare causes of secondary postpartum haemorrhage. Uterine artery embolization was successfully used as modality of treatment.

The development of pseudoaneurysm is a complication of vascular injury resulting from inflammation, trauma, or iatrogenic causes such as surgical procedures, percutaneous biopsy or drainage ${ }^{7}$.The efficacy and safety of selective embolization of uterine arteries was evaluated by Pelage et al ${ }^{8}$, in women with delayed secondary postpartum 
haemorrhage. Out of 14 women, pseudoaneurysm of the uterine artery was found in 2 women. Immediate resolution of external bleeding was observed after embolization. No complications were noted in this study. Other authors have described complications, including muscle pain, neurologic damage, bladder necrosis and vesicovaginal fistula. Angiographic embolization has the advantages of decreased morbidity, ability to localize the bleeding site and provide a more distal occlusion than surgical ligation and preservation of future fertility compared to hysterectomy ${ }^{9}$.In a cohort study by Salomon et al ${ }^{10}$, embolisation was found to be a safe procedure that does not impair menstruation and fertility. They noted the possibility of recurrence of severe postpartum haemorrhage in subsequent pregnancies which did not occur in our case.

\section{Conclusion}

It is important to be aware that endometriosis is an important risk factor for spontaneous haemoperitoneum in pregnancy especially in cases of IVF. Prompt intervention is needed if patient is hemodynamically unstable. Uterine artery embolisation can be successfully used in cases of hemodynamic stability and successful pregnancy could be achieved after this procedure.

\section{Conflict of interest: None. Disclaimer: Nil.}

\section{References}

1.Roche M, Ibarrola M, Lamberto N, Larrañaga C, Garcia $\mathrm{M}$. Spontaneous hemoperitoneum in a twin pregnancy complicated by endometriosis. The Journal of MaternalFetal and Neonatal Medicine. 2008; 21(12): 924-26.

2.Moreira A, Reynolds A, Baptista P, Costa A, Bernardes J. Case report: intra-partum utero-ovarian vessels rupture. Archives of Gynecology and Obstetrics. 2008; 279(4): 583-85.

3.Diaz-Murillo R, Tobias-Gonzalez P, Lopez-Magallon S, Magdaleno-Dans F, Bartha J. Spontaneous hemoperitoneum due to rupture of uterine varicose veins during Labor successfully treated by percutaneous embolization. Case Reports in Obstetrics andGynecology: 2014; doi: 10.1155/2014/580384.

4.Brosens I, Fusi L, Brosens J. Endometriosis is a risk factor for spontaneous hemoperitoneum during pregnancy. Fertility and Sterility. 2009; 92(4): 1243-45.

5.Brosens I, Lier M, Mijatovic V, Habiba M, Benagiano G. Severe spontaneous hemoperitoneum in pregnancy may be linked to in vitro fertilization in patients with endometriosis: a systematic review. Fertility and Sterility.2016; 106 (3):692-703.

6.Leone Roberti Maggiore U, Ferrero S, Mangili G, Bergamini A, Inversetti A, Giorgione V et al. A systematic review on endometriosis during pregnancy: diagnosis, misdiagnosis, complications and outcomes. Human Reproduction Update. 2015; 22(1):70-103.

7.Dasari P, Maurya D, Mascarenhas M. Uterine artery pseudoaneurysm: a rare cause of secondary postpartum haemorrhage following caesarean section. BMJ Case Reports.2011; doi: 10.1136/bcr.01.2001.3709. [PubMed] [PMC free article]

8.Pelage J, Soyer P, Repiquet D, Herbreteau D, Le Dref $\mathrm{O}$, Houdart E et al . Secondary Postpartum Hemorrhage: Treatment with Selective Arterial Embolization. Radiology. 1999; 212 (2): 385-89.

9.Nanjundan P, Rohilla M, Raveendran A, Jain V, Khandelwal N. Pseudoaneurysm of uterine artery: A rare cause of secondary postpartum hemorrhage, managed with uterine artery embolisation. Journal of Clinical Imaging Science.2011; 1:14.

10.Salomon LJ, de Tayrac R, Castaigne-Meary V, Audibert F, Musset D, Ciorascu R. Fertility and pregnancy outcome following pelvic arterial embolization for severe post-partum haemorrhage. A cohort study. Human Reproduction.2003; 18:849-52.

Daksha Bhobe ${ }^{1}$, Eki Emovon ${ }^{2}$

${ }^{1}$ Specialty Doctor Department of Obstetrics \& Gynaecology, Doncaster and Bassetlaw Hospitals NHS Foundation Trust; ${ }^{2}$ Consultant, Department of Obstetrics \& Gynaecology, Doncaster and Bassetlaw Hospitals NHS Foundation Trust Armthorpe Road, Doncaster, UK, DN2 5LT. 